\title{
SMART SOLUTIONS FOR RFID BASED INVENTORY MANAGEMENT SYSTEMS: A SURVEY
}

\author{
ALI ALWADI* AMJAD GAWANMEH, SAZIA PARVIN\$, AND JAMAL N. AL-KARAKI
}

\begin{abstract}
This article is a survey of the latest technologies, algorithms and state of the art localization techniques that can be used to serve as Internet of Things communication protocol by automating an RFID system. There is a lack of a reliable and up-todate reference that can help inventory management systems developers and operators to enhance the management system efficiency, maximize the productivity, and minimize the material loss. Several low cost IoT devices and associated technologies, such as Radio Frequency Identification system, are widely used today in several applications, including educational, transportation, animal tracking, inventory object tracking, and so many others. In this paper, we present a survey of the state-of-the-art technologies, algorithms, and techniques used in smart Radio Frequency Identification systems based inventory systems. We first outline the design challenges for RFID-based inventory management systems followed by a comprehensive survey of various RFID technologies, RFID types, and RFID architectures. In addition, the latest researches in the RFID infrastructure and middle wares are evaluated. This includes passive RFID Tags, RFID Antennas, RFID middleware, and the RFID Reader. Finally, the paper presents the advantages and performance issues of different techniques in passive RFID, and investigates the collision and anti-collision algorithms for these types of applications.
\end{abstract}

Key words: Inventory Management System, Supply Chain, Smart Solutions, RFID, radio frequency identification, Internet of Things, IoT

AMS subject classifications. 90B05, 68M11

1. Introduction. The recent civilization has forced a major expansion of cities around the world, which resulted in a continuous society and economic growth. This has driven the demand for mobility and automation of manual processes by users in order to make them smart, which will enhance the quality of life. Technology is being deployed in several infrastructures that are very complex and limited by its geographical location [46]. This resulted in several challenges in the design and implementation of devices to be used in such areas.

Mobilizing infrastructure requires a high level of balance between applying service solutions in order to meet the imperative mobility demands and to avoid a future mobility collapse. As a result, mobility related data must be gathered in order to be used to facilitate the public transport use, control traffic, connect emergency services with other health and the executive authorities, facilitate library processes and many other purposes which are too many to be enumerated in this paper [46, 34]. The engine that provides an environment to exchange mobility data between services is Internet of Things (IoT).

IoT can be simply defined in the scope of this article as a network that connects real-life objects into one network, using intelligent network devices, in order to exchange a specific set of information, to form an informational network that is integrated, based on standard communication protocols, Radio Frequency Identification (RFID) for instance. While there is no common definition for IoT, as it depends on the context of the application. In the context of this paper, it can be conceptually defined as a dynamic global network infrastructure with self-configuring capabilities based on standard and interoperable communication protocols. As physical and virtual "things" have identities, physical attributes, and virtual personalities and are seamlessly integrated into the information network. It can be seen as a new paradigm that includes a wide range of new technologies [4, 20, 17, 21, 3, 41].

RFID is the most common underlying communication protocol that serves the IoT concept, by connecting everyday objects using an RFID network. RF Identification is an object identification mechanism in which wireless communication technology is used as a communication protocol, which uses Radio Frequency (RF) signal for the communication between the RFID antenna and the tag. The physical objects are made active

*School of Engineering, Auckland University of Technology Auckland, New Zealand. ((ali.ab.alwadi@gmail.com)

Department of Electrical and Computer Engineering, Khalifa University, UAE. and Department of Electrical and Computer Engineering, Concordia University, Montreal, Canada. (amjad.gawanmeh@kustar.ac.ae).

$\S$ Melbourne Polytechnic, Victoria, Australia and University of New South Wales, Canberra, Australia. (saziaparvin@ melbournepolytechnic.edu.au).

$\sharp$ Department of Information Security Engineering Technology, Abu Dhabi Polytechnic, Abu Dhabi, UAE, and Dept. of Computer Engineering, The Hashemite University, Zarka 13115, Jordan. (jamal.alkaraki@adpoly.ac.ae). 
network components by attaching a network interface to these objects. Using these interfaces, services will be able to interact with these "smart things/objects" that will provide the necessary link via the internet, to query and change their state, and retrieve any information associated with them, taking into security and privacy issues which will be solved by introducing the appropriate algorithms $[5,37,3]$. In our daily life, retrieving information from the connected things in the network will result in a huge data, which will require instant processing and analysis.

The revolution of cloud computing and IoT provides an opportunity to make technology touches unprecedented areas of our daily life. However, IoT consists of a number of heterogeneous devices and applications which their exchange information can differ in the format, size, and sometimes the protocol. That makes it hard for traditional cloud computing servers to recognize and process the exchanged information between the servers and the objects. Therefore, an improvement on the cloud computing servers is required to efficiently schedule and allocate IoT requests [36].

Cloud computing is a newly developed trend of the use of information technology (IT), which virtualizes the way computing resources are used and managed through new techniques. Cloud has shifted the IT industry to a new era with plenty of opportunities, induced a revolution in IT applications, and significantly influenced the way businesses use IT to create competitive advantaged. Commercially, the majority of the IT solutions providers recognize IT as a strategically crucial resource that contributed in the latest revolution, by bringing substantial benefits, such as cost saving, scalability, mobile storage, ease of access, better security, energy saving, and environment benefits [11,44]. Cloud computing is a shared IT infrastructure where computing resources are scattered but linked together through the internet into a large pool of computing resources. That could automatically adjust the allocation of computing resources as the need for computing service fluctuates, resulting in higher utilization and productivity $[11,25]$.

Inventory management is a critical component of efficient supply chain management that is vital for success in a modern computing infrastructure. It is well-known that supply chain inventory management decisions depend on inventory data gathered from automated or manual control systems [49].

There are some complementary attributes between cloud computing and IoT. For instance, the centralized nature of cloud and the pervasive nature of IoT, the virtual nature of cloud supplements the real-world things in IoT, the ability to store a huge amount of information supplements the limited storage space for IoT [35].

In the next section, the concepts of IoT and Cloud will be briefly introduced, the relationship between them, and how IoT contributed in automating cloud based inventory management systems. In section 3, RFID Principles and Categories is explained. Section 4 explains possible middleware servers to run inventory management systems. And finally, conclusions and future work is explained in section 5 .

2. Internet of Things and Cloud Systems in Inventory Management. Technological innovation and development has seemingly become the new de facto standard across many research facilities around the world. Technology users are wired in and logged on across the planet, each minute collectively generating or consuming 640 terabytes of data, photos, and videos on their handheld computing devices, tablets, and, more recently, wearable devices. In 2016, more than 5.5 million connected devices are added every day, and IoT is well on its way to involving more than 20.8 billion devices worldwide by 2020 [51].

2.1. IoT in Inventory Management. IoT is creating a new operation environment where each physical asset is individually identified with an ID, intelligently connected into a network, and digitally visible to the whole supply chain at a by-unit level and in real time [7]. IoT underlies almost all the emerging technologies used today. Mobile phones are the most prevalent example of popular technology. Driverless cars, is another application of this technology, which works through interconnectivity between the road, online maps, and other connected data, including weather predictions and traffic reports [51]. The BodyGuardian is a wearable sensor system that can remotely read a patient's biometrics (electrocardiogram, heart rate, respiration rate and activity level), sending data to the patient's physician and allowing users to go about their daily lives outside of a clinic facility [3, 51].

Similarly, by linking machines, products, people, and supply chain members, IoT provides a new environment for supply chain managers to manage and control inventory resources using applications that run on middleware servers [7]. The feasibility and flexibility of the architecture of this supply chain was proposed through a detailed implementation that uses wireless sensor networks and web services to introduce the use 
of the smart object framework, these networks encapsulate RFID, sensor technologies, embedded object logic, object ad-hoc networking, and internet-based information infrastructure to realize the real-time monitoring of the flow of goods through a supply chain [54]. The implementation of RFID in the supply chain was employed to achieve real-time inventory monitoring and information sharing, such approach can help the system attain high environmental and economic benefits [54].

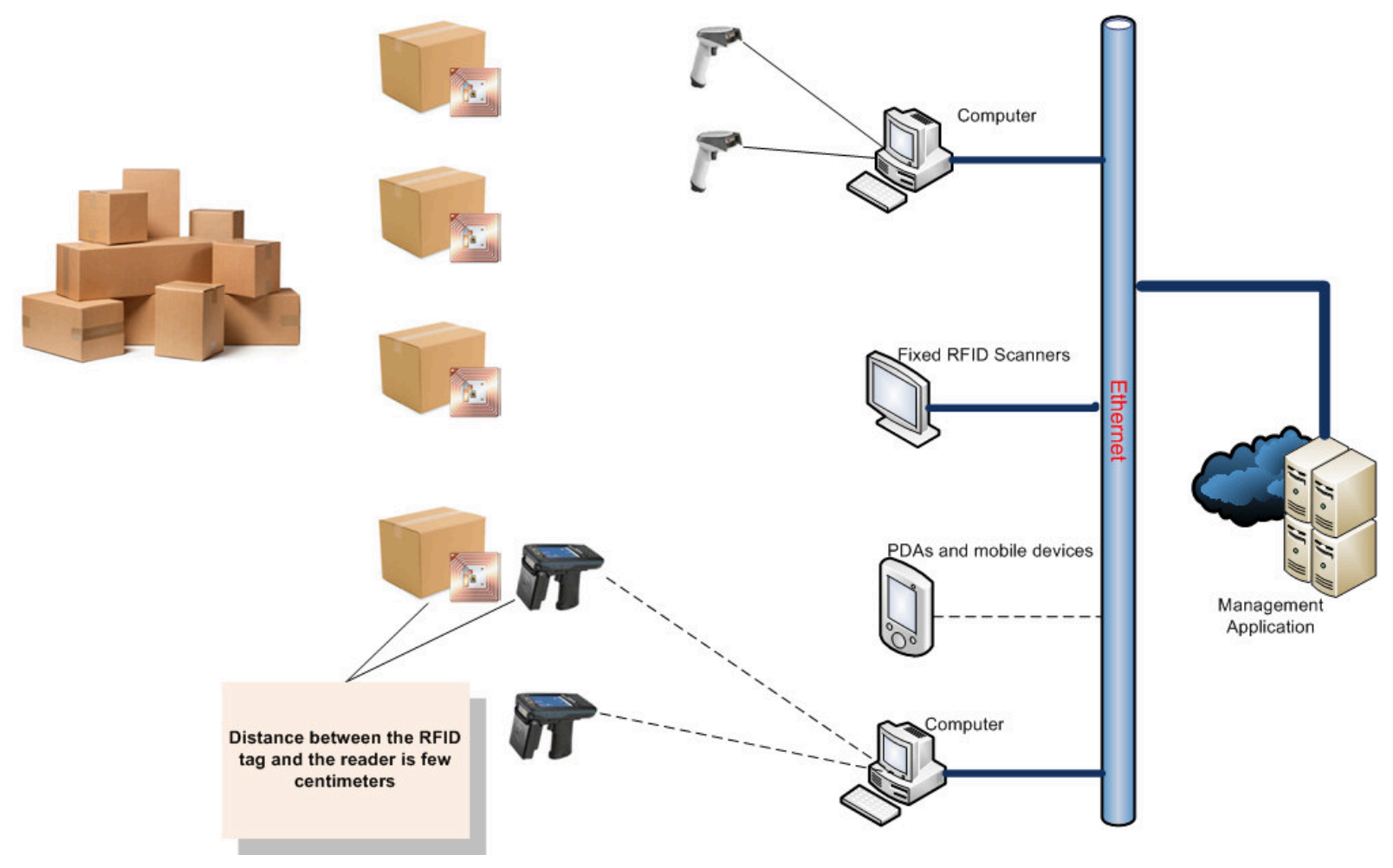

FIG. 2.1. Inventory System Architecture

The network of inventory objects is shown in Figure 2.1. By attaching an RFID tag to every item in the inventory, the tagged object becomes part of the network. The inventory administrator uses the handheld RFID readers to scan the objects, handheld readers nowadays can be wireless and smart with processing capabilities, they are connected to the middleware using any of the various computer cables or wireless using Wifi communication protocol IEEE 802.11. The reading distance between the reader and the tags cannot exceed a few centimetres, and both ends must be held appropriately to achieve the required polarization angle. The cloud is serving as the middleware provider for the management system.

RFID and associated technology extensive use of in several applications, including educational, transportation, animal tracking, inventory object tracking, and several industrial applications. This have made it recognized as a standard for IoT applications. Two main reasons contributed in this standardization, the increasing demand on this technology and the availability of RFID tags at very low prices. As a result, the cycle of manufacturing RFID-related products has speeded up lately [33].

2.2. Cloud Systems in Inventory Management. Cloud computing is a shared IT infrastructure where computing resources are scattered but linked together through the Internet into a large pool of computing resources and that could automatically adjust the allocation of computing resources as the need for computing service fluctuates $[11,15]$. This results in a model for provisioning processes, applications and services that is potentially cost-efficient. In addition, it can make IT management simpler and increase business responsiveness as well [15].

The evolution of Cloud Computing concept has been driven by the accelerated shift in the computing 
paradigm in the past half century. Initially, computing power existed mainly in mainframes shared by users using terminals. Standalone personal computers had their share of this revolution, they came powerful enough to process the users' tasks. This has encouraged another phase of this evolution. Users having powerful computers can now exchange information, and share processing power using computer networks. The next phase of the evolution is the internet, a network of local networks connected to form a more global network to utilise remote applications and resources. Finally, the concept of distributed systems came along, which is an computerized grid that facilitates sharing the computing power and storage across the network components. This forms the backbone of cloud computing, as cloud enables utilization of the available resources in a scalable and simple form [15], unlike a mainframe is a physical machine that offers finite computing power. Unlike mainframe, a cloud represents all possible resources on the Internet, offering infinite power and capacity.

Cloud computing applies distributed arithmetic techniques, which allows developers to more easily develop application services. It can automatically manage large numbers of computers for task and storage distribution. In 2003, the NSF invested USD 8.3 million to support the network virtualization and cloud computing VGrADS, was launched by the top U.S. seven colleges, which was when the research on cloud computing was born [14, 32].

Cloud plays a key role in RFID identification process through:

- Managing the hardware resources in the RFID network efficiently, RFID readers, antennas, and tags.

- Managing the information flow from/to the inventory repository regardless of the type of the database engine or the platform used.

- Running the inventory management software, and provide the users with a platform-independent, stable, and robust real-time management software. The user must interactively be able to access the management system, add, modify or delete inventory resources, and the changes must take effect instantaneously.

Cloud computing is organized into three standard service models [19], see Figure 2.2:

- Infrastructure as a Service (IaaS): The cloud vendor provides the servers (such as processing capability), storage (such as replication, backup, and archiving), and connectivity domains (such as firewalls and load balancing).

- Platform as a Service (PaaS): Describes a model in which the cloud vendor provides the platform that allows creation and deployment of applications and services the organization accesses through the Internet.

- Software as a Service (SaaS): The cloud vendor has complete control over the application, including capabilities, updates, and maintenance. The user is provided with a secure and versatile service accessible via the internet.

3. RFID Principles and Categories. RFID can be used to locate and track items in warehouses and during the entire shipping route as well [53]. Furthermore, RFID has extensive applications such as transportation and logistics, asset tracking, inventory management, and healthcare [58], object identification and tracking [56], security in airports, malls and military bases [31, 48, 53, 55, 56, 58], and so many others. In this article, an overview of the RFID technology and its history is first provided, then RFID types and architectures are explained. In addition, the article analyzes the latest developments of RFID infrastructure and middleware architectures, starting from passive RFID Tags, RFID Antennas, RFID middleware, and the RFID Reader. Finally collision, and anti-collision algorithms in RFID will be explained.

RFID networks can be divided into three main categories based on the tag types: Passive RFID, active RFID, and semi-passive RFID Tags. All three types contain integrated circuit that store tag information [33]. Passive tags are called passive because of the lack of power source, they depend on the energy transmitted from the reader antenna radio frequency signal to power up the tag. On the other hand, active tags contain a small battery that provides the integrated circuit with the power required to operate the tag circuitry, which is why active tags have better coverage, enhanced storage capacity, and they are often programmable. While passive tags on the other hand, have smaller read range, less memory capacity and they are usually programmed once $[33,40,50]$.

Passive UHF RFID is being widely used in order to identify and track several types of inventory items as illustrated in Figure 3.1. Before moving to the technical aspects, one technical term that is tightly bound to RFID needs to be explained, which is Load Modulation. For the antenna to transfer data, a basic system of 


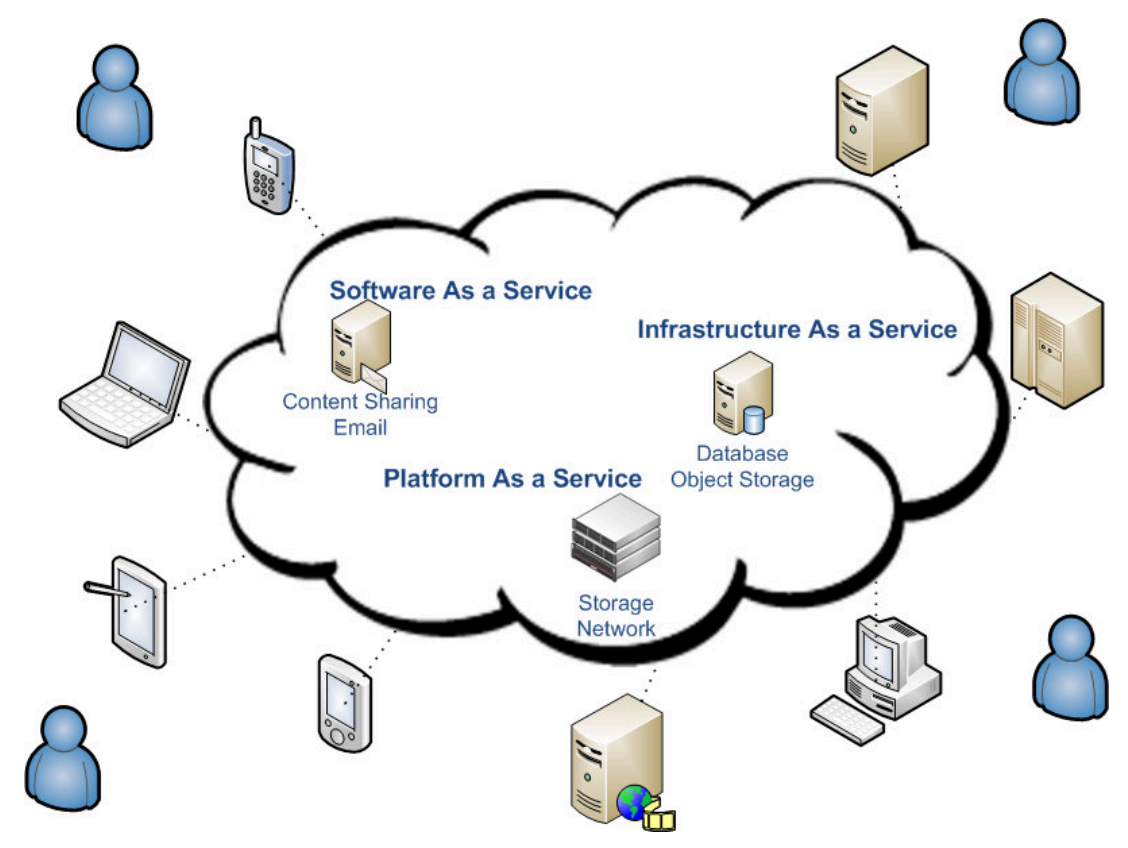

FIG. 2.2. Inventory System Architecture

modulation resistance, in parallel with the tag antenna is switched on and off at a rate governed by the signal data, the receiver on the other side will need to strip out the carrier signal [45]. The tag's antenna captures energy from the transmitted signal, and transfers the modulated signal which contains the tag ID. The receiver circuit is often called transceiver, it is responsible of managing the tag's power in general. In other words, it captures the energy received by the tag's antenna, and release it to feed the tag components. The receiver circuit has gained a considerable amount of research during the recent years $[4,10]$. The memory chip contains a preprogrammed unique Identifier [4, 50].

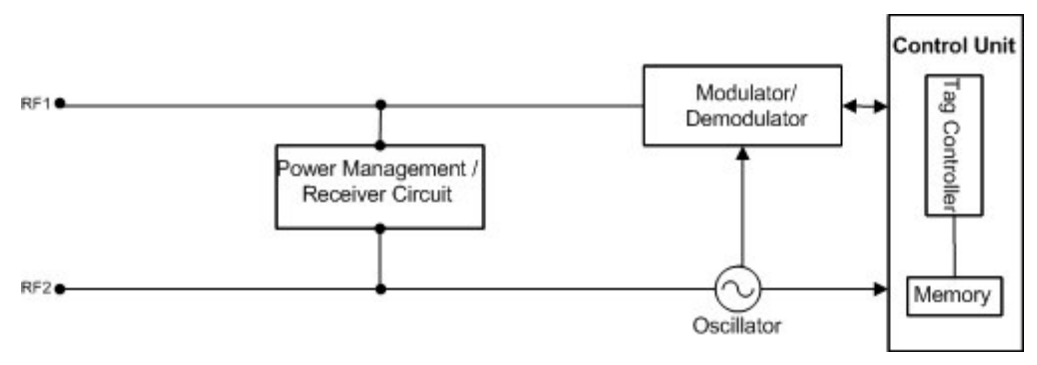

FIG. 3.1. Passive RFID Tag Components [4]

RFID tags are usually classified based on the ElectroMagnetic (EM) wave that is used to transfer the signal. The first category is Near-Field, which depends on the direct magnetic induction of the transmitted signal. The second type is Far-Field, which depends on capturing the energy release from the electromagnetic wave, which is a result of the transmitted radio wave from the antenna. The principle of the communication protocol in both techniques is similar. Both of them utilize the electromagnetic field attached to the RF signal to transfer information, the power transferred normally varies between $10 \mathrm{~mW}$ and $1 \mathrm{~mW}$ [50]. Far-field is generally used in applications where a long read range is needed. Near-field RFID is generally used to track tags in areas contain metallic barriers $[18,38,4]$. Inductive coupling technique is preferred in most near-field RFID applications mainly for one reason, the flexibility of using the load modulation techniques to transfer signals between the tag and the antenna and vice versa [39]. On the other hand, the electromagnetic field in far-field is radiative in 
TABLE 3.1

Characteristics of Near-Field Vs. Far-Field

\begin{tabular}{|l||l|l|}
\hline Property & Near-Field & Far-Field \\
\hline \hline Read Range & $5 \mathrm{~mm}$ - 10cm (Antenna Dependent) & Max. 22.1m \\
Modulation & Load Modulation using capacitive coupling & Electromagnetic radiation \\
Electromagnetic Signal & Radiative signal & Radiative signal \\
Reader Antenna & Small, Omnidirectional & $\begin{array}{l}\text { Resonant, directional. Small an- } \\
\text { tenna size/high frequencies }\end{array}$ \\
Usage & Metal or liquid surrounded objects & $\begin{array}{l}\text { When long reading range is re- } \\
\text { quired }\end{array}$ \\
\hline
\end{tabular}

nature, making the choice of modulation techniques must narrower.

Coupling captures EM energy at a tag's antenna as a voltage disturbance. Part of the energy reaches a tag's antenna is reflected back due to an impedance mismatch between the antenna and the load circuit. The amount of reflected energy vary depending on the impedance of the antenna. This is called Backscattering [28]. The Table 3.1 clarifies the characteristics of Near-field versus Far-field and their usage.

3.1. Near Field RFID. Near-Field is a technology where magnetic coupling is used for communication between the reader and tag. In this technology, the modulated RF signal is transmitted through magnetic induction, where the reader passes an AC current through a coil inside the reader, which generates a corresponding alternating magnetic field. A capacitor is used to rectify this voltage, a reservoir of charge accumulates, which is enough to power the tag chip [33]. Figure 3.2 illustrates Near Field RFID technology.

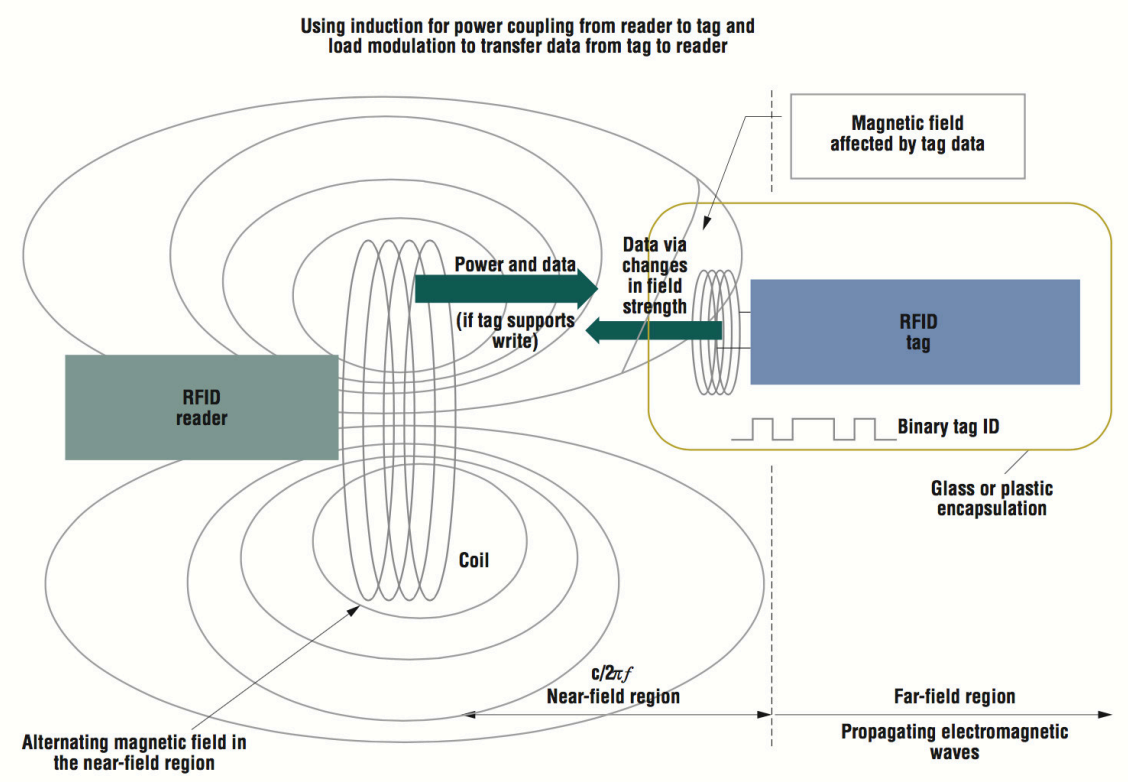

FIG. 3.2. Load Modulation in NearField [50]

Near-field coupling tags use low carrier frequencies. The two most commonly used are $128 \mathrm{kHz}$ and 13.56 $\mathrm{MHz}$. For instance, the maximum read distances are $372 \mathrm{~m}$ for $128 \mathrm{kHz}$ and $3.5 \mathrm{~m}$ for $13.56 \mathrm{MHz}$. The disadvantages of using low frequencies in near-field are [4, 9]:

- To capture the RF signal, an antenna with a large coil is needed, as the power generated from the antenna is very low.

- The radiated power decreases heavily as distance increases. 
- A low data rate resulted from the low bandwidth used.

Due to its design simplicity, Near-Field system was one of the first RFID techniques to be used for the implementation of RFID tags, in particular, passive ones. This technology, however, suffers from physical limitations due to the need of very close contact between the tag and the antenna in order to produce coupling. The range of magnetic induction that can be used is highly dependent on the tag's frequency. As the frequency of operation increases, the distance over which near-field coupling can operate decreases. The energy available for induction is another limitation as a function of distance from the reader coil. As distance increases, the magnetic field drops off at a factor, which can be calculated in the equation $[33,58]$ :

$$
F=\frac{1}{r^{3}}
$$

$F$ is the drop factor, and $r$ is the distance between the antenna and the tag. Since applications require maintaining a fixed read rate, tags with with various polarization poles must be detectable, as a result, more tag requirements have arouse, for instance higher read rate and higher radio frequency. These requirements have resulted in using far-field in passive RFID identification [33].

Power path transmission loss in near field is defined in literature as:

$$
P_{r}=K \frac{1}{r^{\alpha}} P_{t}
$$

where $P_{r}$ is the received power, $K$ is constant that depends on antenna gain and wavelength, $P_{t}$ is the transmitted power. In addition, for near field $\alpha$ can be considered as: $2<\alpha<4$.

The read range for near-field coupling is narrow. The conventional solid-line loop antennas with one operating wavelength can produce uneven magnetic field over the interrogation area, since phase-inversion occurs across the loop conduction material, and therefore the electrical current reaches zero as it passes the antenna material. The reliability of RFID tag detection depends on the strength of the magnetic field. Hence, the relatively weak field in far end regions of the interrogation zone results in degradation of the detection reliability. As a result, the reader antenna must be redesigned to accommodate a larger antenna in order to maximize the coverage area. For instance, a tag antenna of size $150 \times 150 \mathrm{~mm}^{2}$ can compensate the shortage in the read distance. $[33,58]$.

3.2. Far Field RFID. To transfer signals, Far-field system utilizes the electromagnetic field that results from the emittance of the radio frequency signal. Far-field systems operate at the UHF region of the spectrum, normally between $840 \mathrm{MHz}$ and $960 \mathrm{MHz}$, or microwave frequencies, between $2.45 \mathrm{GHz}$ and $24 \mathrm{GHz}$ [8]. A dipole antenna that is imbedded in the reader antenna transmits EM waves to the tag. The EM wave triggers an alternating potential difference once received by the tag's small dipole antenna, which appears across the antenna circuit, this forms an alternative current that is passed through to a diode which is also linked to a capacitor, which will then be rectified as an energy that the tag uses to power up the chip. However, unlike inductive coupling, tags are beyond the range of the reader's EM wave area, and information cannot be transmitted back to the reader using load modulation [50].

A far-field system's range has some limitations, which can be summarized:

1. The amount of energy received by the tag.

2. The sensitivity of the reader's antenna to the signal received from the tag.

As the electromagnetic wage travels, attenuation occurs on both sides of communication, between the antenna and the tag, and the response signal from the tag. The received signal is expected to be weak. The returning energy from the tag is $[33,58]$ :

$$
F=\frac{1}{r^{4}}
$$

where $r$ is the distance.

Nowadays, the semiconductor revolution has helped in reducing the size of the electronic materials in general, which helped in decreasing the power necessary for these components to consume in order to operate as expected, RFID tag has had its share of this innovation, power consumption continues to decrease, which helped 
in expanding the RFID operations and usages. In addition, low cost customized RFID tags can be designed and manufactured. These can efficiently read from a wider distance which sometimes can reach up to 6 meters with power usage as low as $100 \mathrm{dBm}[50]$.

3.3. RFID based Communications. RFID systems operate in a different range of the spectrum depending on the antenna and tag design, which can vary from $100 \mathrm{kHz}$ to $5.8 \mathrm{GHz}$. The underlying technology requires the RFID systems to operate in the UHF frequencies occupying the ISM bands $860-960 \mathrm{MHz}$, according to frequency band allocation in each country. The read range offered by UHF RFID makes this frequency the most attractive for use by supply chain management. The allocated band at ultra-high frequency (UHF) ranges from $860 \mathrm{MHz}$ to $5.8 \mathrm{GHz}[33,16,13]$.

3.4. RFID Tag. RFID tags are passive, active or semi-passive. The term, passive or active is referred to the power source that the tag uses to extract the required energy to operate. Passive tags retrieve the energy from the electromagnetic signal transmitted from the reader's antenna. The tag retrieves the required energy from the interrogating wave or from a modern antennas have a dedicated port that transmit constant signal to power up tags in the range. The second type of tags is active tag, which have internal batteries as a power source, to enhance the reading range. Passive tags have the lowest price in terms of manufacturing cost, they do not require maintenance, more compact and lighter [33, 16].

Semi-passive tags are similar to the passive tags in the way they operate, they use backscattering technique to respond to the reader. The main difference is that semi-passive tags have batteries to power the chips that are embedded in the tag, which is used in conjunction with integrated electronic components such as sensors. Semi-passive tags have almost the same characteristics of the passive tag (reading range, operating frequency) [13].

RFID tags contain RF transponders with digital memory chips that are uniquely identified. The antenna packaged with a transceiver and decoder, emits a signal activating the tag, refer to the Figure 3.3 [4, 33]. A brief description of each RFID tag component is mentioned below [52]:

1. RF Interface The radioactive component of the tag, which performs the following:

- Supply RFID transponders with power by generating the required energy.

- Modulating the signal in preparation for the transponder to transmit.

- Reception and demodulation of signals received by the transponders.

2. Control Unit As the name implies, this part of the tag controls the tag operation, by performing the following functionalities:

- Execution of the application software commands.

- Signal interpretation.

- Controls the communication with the transponder.

- Anti-collision, and encryption can be implemented in the control unit.

- Transponder-reader authentication.

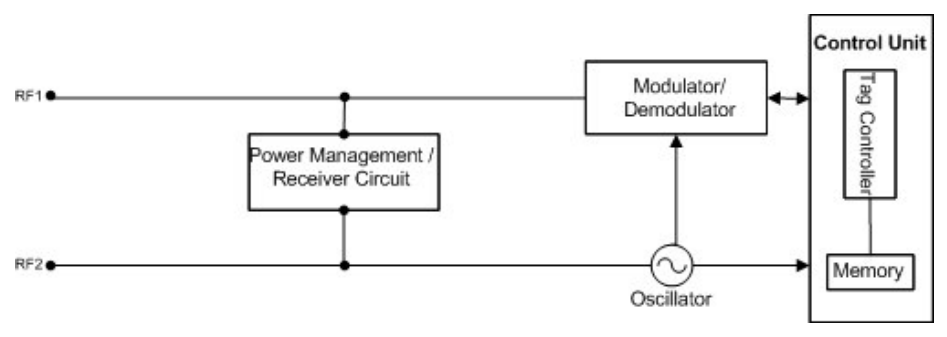

FIG. 3.3. Block Diagram for Passive RFID Structure

The capacity of the tag varies depending on the specifications of the vendor. It is very common ti find most modern tags with a capacity of up to 2,048 bits of information [33, 52]. This is enough to store the necessary information required about library items. 
3.5. RFID Reader Antenna. As explained earlier, RFID reader antenna transmits signals as electromagnetic wave called radio frequency wave. Antennas can be classified into two main types based on the characteristics of the transmitted signal [26]:

- Linear Polarization: The electromagnetic wave propagates in a single direction, vertically or horizontally, depending on the orientation. These antennas are also referred to as Dipole antennas. This type of antennas is recommended to use when the tag has fixed orientation. Therefore, it is commonly used in hand-held readers in warehouses and inventories. In order for this antenna to work, the reader and the tag must be aligned together. This is a major drawback of this type of tags.

- Circular Polarisation: The electromagnetic wave covers the two planes during propagation, in a circle-like motion that resembles the motion of a screw, which makes this type more efficient than the linear polarization for two main reasons: The wide coverage area, and the tag orientation does not have to be inline with the antenna in order to make a successful read. One disadvantage of this antenna is energy loss, since the majority of the energy in the transmitted signal lies within the first few waves, as illustrated in Figure 3.3.

RFID antennas have also a commercial classification that divides antennas into two main groups, based on the number of ports that the antennas have [26]:

- Monostatic Circular: This antenna is equipped with a single port, which is used for transmission and receiving of the RFID signals.

- Bistatic Circular: This antenna has a dual ports, one for transmission and one for receiving signals. This type of antennas is more common, but it is more expensive.

Both antennas can be manufactured with a Listen Before Talk (LBT) port, which is a dedicated port that listens for signals before sending RFID signals. The read range for both types depends on [29]:

- The available power at the reader/interrogator to communicate with the tags.

- The quality of the tag's circuitry, and its ability to capture the released energy to power the tag's components.

- The environmental conditions and structures, as the operation frequency increases, the probability of the signal being obstructed by metal or a wall is higher.

The released wave from an antenna propagates in space in a circular motion, its strength diminishes as the traveled distance increases. The design of the antenna determines the shape of the delivered wave, as a result the read range and the positive identification of tags are also affected by the distance between the reader and the tag, as well as the orientation of the antenna. In an ideal situation, free of obstructions or absorption objects, the signal strength decay in inverse proportion to the cube of the distance (refer to equation 2) [22]. When an antenna transmits a power signal, regardless if it was a constant power signal or a regular RF signal, the tags in the range are powered up and will reply with their identification signal, for a large number of tags, this will cause an issue, it is called collision, which heavily affects the efficiency of identification.

3.6. Collision and Anti-Collision Algorithms. Collision is an undesired interference that occurs between radio signals initiated from two or more radio frequency components that lie within the frequency range of each other. Normally, an inventory system equipped with passive RFID contains a high density of RFID equipment that transmit signals around, collision happens often when two or more radio active components reply to the reader simultaneously. Collision is a critical terminology in RFID networks for the following reason, when the reader antenna transmits the power up signal, which normally happens by sending the read signal, all tags within the reach of reader signal will detect and respond to it with their modulated signals. Collision occurs here as a result of the antenna receiving tens and sometimes hundreds of response signals from the tags. An algorithm is required to "detect" or organize each tag's signal and marshal the signals in orderly manner without interference of signals from other antennas or tags, applying anti-collision algorithms enhances the network throughput by reducing the time required to perform the identification. Various anti-collision algorithms have been proposed, the main two algorithms: (a) ALOHA-based algorithms, the frequency range is divided into time slots, each time slot is assigned to one tag only. (b) Tree-based algorithms that group the tags into a tree with branches that contain subsets. The identification of tags is achieved by iterating through the tree branches [4].

ALOHA is an algorithm built based on reducing the probability of tag collision by assigning time slots to 
tags. It utilizes Time Division Multiple Access (TDMA) to implement a collision resolution [30]. This document focuses on ALOHA-based algorithms for two main reasons, they work well with Read-Only tags, in other words, they don't require the tag to be reprogrammed to hold extra information. The second reason is simply because they are the most frequently used anti-collision techniques. Dynamic Frame slotted Aloha (DFS-ALOHA) in particular will be discussed in this article. By anticipating the probability of collision to estimate the number of tags in the interrogation zone, the algorithm determines the appropriate frame size depending on the number of tags. To avoid collision, DFS-ALOHA algorithm allocates a random frame for each tag to transmit data in. The bandwidth is divided into frames, and the frames are divided into slots. The system efficiency depends heavily on numerous factors: The amount of tags, the distribution of tags around the antenna, and the estimated frame size $[23,47,57]$. However, ALOHA-based algorithms have one disadvantage, "Starvation". A tag may not be allocated a time slot to use to load its signal, which makes it undetectable by the surrounding antenna(s), as a result, the tag may not be detected for a a period of time, and sometimes forever [12].

Tree-based algorithms are deterministic, they use a search tree to identify tags that fall in the frequency range of the antenna by classifying the tags in the interrogation zone into a tree. The algorithm iterates through the branches until all tags have been identified. Tree-based algorithms are divided into three types: Binary Tree (BT), Query Tree (QT), and finally Binary and Query Tree, which is a combination of both [16, 24, 27]. QT uses tag IDs to group the tags into subsets, then the system iterates through the subsets by dividing all subsets into groups that contain a set of two. The efficiency of the identification process is affected by the distribution of tags in the system. BT uses randomly generated numbers to identify the tag groups, which requires reprogrammable tag memory to store the assigned number in order to make this algorithm more efficient, however, in case of passive RFID reprogramming the tag chips are not programmable [6].

4. Middleware for Inventory Management. The Middleware is a software that facilitates the exchange of information between the applications, repositories and the integrated hardware. It is an engine that deploys the binaries and application executable files which are responsible of managing the flow of data between the antennas, readers and the management application. The middleware application server operates the integrated components, and manages the flow of information between the application and the peripherals. It also supports readers with the connectivity, context filtering and message routing, and integration with the server interfaces. To achieve successful tag identification, the middleware must achieve the following [6]:

1. Real-time processing of transaction events from the hardware components.

2. The middleware must provide a common interface to access different kinds of hardware offering different features.

RFID middleware is composed of four main layers [6]:

- Reader Interface: This layer is close to the RFID hardware. It manages the flow of information with the RFID hardware components. It manages the hardware interface related parameters, for instance reader protocol, and reader interface.

- Data Processing and Storage: Processing the data received from the readers, and storing the transformed information into a repository.

- Application Interface: Providing the user with user friendly interface required to control the RFID Middleware application and configure the components attached to it.

4.1. IBM WebSphere Premises Server. WebSphere Premises Server is a framework that is based on the foundation of a service-oriented architecture (SOA). It can efficiently deliver several services though which sensor integration solutions are supported. As a result, this server can provide a robust, flexible, and scalable platform for capturing application-driven information from sensor data. This platform can be used for integrating new sensor data, identifying the relevant application events using situational event processing, and then integrating and acting upon those events with the deployed SOA application processes, which are designed to handle those events, process them, and store them in the designated repository [15]. The middleware application must be built with an integrated sensor-based functionality in order to retrieve data from sensors, and build business transactions out of these raw input data that can lead into business decisions. WebSphere Premises Server delivers the platform of scalable, reliable end-to-end sensor business solutions by [15]:

- Extending IBM SOA process integration platform to integrate applications with sensors to provide them with sensor data and events allowing system administrators to flexibly deploy applications at runtime 


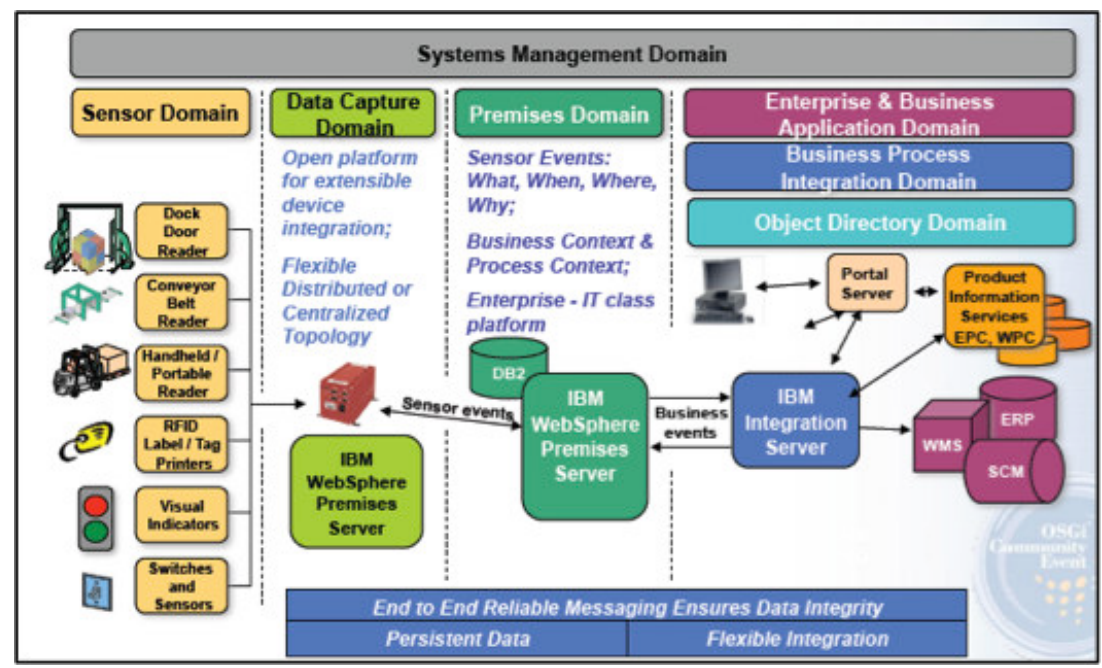

FIG. 4.1. WebSphere Middleware Architecture [15]

without the need to reorganize or restart the server.

- Providing an engine with intelligent business rules to develop identification for complex events from multiple types of sensor data.

- The server is bundled with system features to support real-time location tracking services, including device interfaces for RFID components, core localization processes and a graphical user interface for location visualization.

- Enhancing device driver services, allowing a single core platform to support multiple sensor types including passive RFID, active RFID and sensors.

- Providing an Integrated Development Environment (IDE) friendly platform for integrating sensor devices using customized applications.

- Integration with WebSphere RFID Information Centre to enable the deployed applications to efficiently manage and integrate sensor information with enterprise applications as well as securely share sensor information and events with selected third party applications.

- WebSphere Server stack contains three main components as shown in Figure 4.1: RFID devices, WebSphere Premises Server, and WebSphere Business Integration Server [15].

4.2. Rifidi Edge Server. Rifidi Edge Server is an engine that connects the IoT components with people who use handheld and mobile devices, over the cloud. It is a complete RFID Middleware Platform with built-in integrated development tools to enable the development and deployment of customized RFID applications. The product is an open source alternative to popular RFID platforms such as IBM Premises Server and Microsoft Biztalk RFID. Built on the latest Java OSGI platform and integrated with powerful open source rules engine (Esper). Rifidi Edge has the ability to build complex applications that interact with the most popular RFID and sensor devices available in the market [43].

The most fundamental functionality for Rifidi Edge Server is to gather data from sensors, and deliver them to middleware applications that use the data for processing and storing [42]. The server filters out all the noises and distorted signals that the sensors deliver to the middleware, which is an important factor in the RFID area in order to filter out all the undesired radio signals.

Figure 4.2 contains a high-level description of how data are collected and transferred through the edge server. Sensor and Reader Abstraction layer is the first module that received data from the sensors or any connected RFID component, which normally contains customized programming interfaces to interact with the custom sensors. While these components are normally hardware RFID readers, such as Alien 9800 and Symbol XR400. Data might also be produced by a legacy barcode reader, a database, or even another edge server.

As data are collected from the sensors, they are passed into a high-speed internal message queue system in 


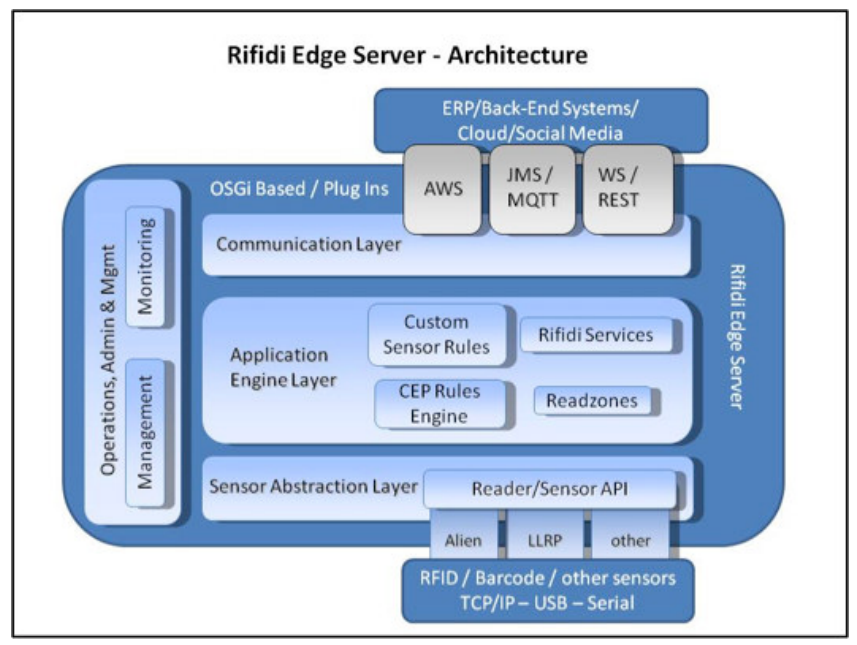

FIG. 4.2. Edge Server data flow

order to be exchanged with other server components. The Application Layer Events (ALE) provides the system with an interface to collect and filter RFID data. Data collection according to ALE rules is achieved by using an event stream processor called Esper [42, 29].

5. Conclusions and Future Work. This article is intended as a survey for the latest available research in Cloud Computing, inventory management middleware servers, and RFID Identification and localization algorithms that can be used to automate an inventory that has a passive RFID infrastructure. As illustrated in Table 5.1, several technologies that can be potential candidates to be used to automate an inventory system using cloud are summarized in this work. This article is a baseline for the system owners and implementers to locate inventory objects (or the Tagged objects) at runtime, without having to replace (or possibly reprogram) the passive RFID tags. Using the proposed algorithms, technologies allow the system owner to achieve the following:

- Automate the object localization, and remotely control the automation process using simple but efficient

TABLE 5.1

Summary of technologies used in smart inventory systems

\begin{tabular}{|l|l|l|l|}
\hline \hline Group & Reference & Technology Used & Problem Addressed \\
\hline \hline IoT & {$[34][46]$} & IoT devices & $\begin{array}{l}\text { Utilizing IoT Concepts in mobilizing } \\
\text { cities }\end{array}$ \\
\hline Hybrid & $\begin{array}{l}{[3][4][5][17][20][21]} \\
{[31][33][37][48][53]} \\
{[54][55][56][58]}\end{array}$ & IoT and RFID & $\begin{array}{l}\text { Using RFID as a communication } \\
\text { protocol for IoT }\end{array}$ \\
\hline Cloud & $\begin{array}{l}{[11][15][19][36][44]} \\
{[51]}\end{array}$ & Cloud Computing and IoT & $\begin{array}{l}\text { Cloud Computing serving IoT appli- } \\
\text { cations }\end{array}$ \\
\hline Inventory & {$[7][14][32][35][49]$} & Inventory Management & $\begin{array}{l}\text { Automating an Inventory Manage- } \\
\text { ment system using IoT concept }\end{array}$ \\
\hline RFID & $\begin{array}{l}{[4][8][10][13][16][33]} \\
{[45][50][52][58]}\end{array}$ & $\begin{array}{l}\text { RFID and Passive RFID } \\
\text { Tag infrastructure }\end{array}$ & $\begin{array}{l}\text { Available RFID types, and the tag } \\
\text { structure and operating frequency of } \\
\text { each type }\end{array}$ \\
\hline Antenna & {$[22][26]$} & RFID Reader Antenna & RFID Antennas and wave types \\
\hline Middleware & {$[6][15][29][42][43]$} & RFID Middleware & $\begin{array}{l}\text { Middleware for Inventory Manage- } \\
\text { ment applications }\end{array}$ \\
\hline \hline
\end{tabular}


management system, which can be accessed anywhere, anytime.

- Dynamically add or remove system resources, RFID objects from the network with having the need to modify the management or the tracking code.

- Implement a fault-tolerant system, which can detect hardware and software failures

Based on the performed survey, there are several issues that can be addressed in order to provide reliable RFID based inventory system, such as automated library system. First, a hardware prototype as well as a simulation software are required, in order to capture all design specifications, including operating frequency, read range, noise factor, energy loss. The simulation results must be recorded and matched against the hardware implementation. Next, a chosen middleware need to be configured and integrated with the workbench in order to start the hardware integration with the RFID readers. New Command line interface must be developed to integrate the middleware server with the reader antennas. Initial simulation results proved that such system will be efficient and reliable for the purpose of automating inventories.

\section{REFERENCES}

[3] A. Al-Fuqaha, M. Guizani, M. Mohammadi, M. Aledhari, And M. Ayyash. Internet of things: A survey on enabling technologies, protocols, and applications. IEEE Communications Surveys Tutorials, 17(4):2347-2376, Fourthquarter 2015.

[4] Ali Alwadi, Jeff Kilby, Amjad Gawanmeh, and Hakilo Sabit. Radio frequency identification technology in libraries. International Journal of Engineering Research and Science, 2(11):65-77, November 2016.

[5] R. E. A. ANEE AND N. C. KARMAKAR. Chipless rfid tag localization. IEEE Transactions on Microwave Theory and Techniques, 61(11):4008-4017, Nov 2013.

[6] R. Bhattacharyya, C. Floerkemeier, and S. Sarma. Low-cost, ubiquitous rfid-tag-antenna-based sensing. Proceedings of the IEEE, 98(9):1593-1600, Sept 2010.

[7] Li Bo AND Li YulOng. Internet of things drives supply chain innovation: A research framework. International Journal of Organizational Innovation, 9(3):71 - 92, 2017.

[8] D. Caldwell-Stone. Rfid in libraries. Library technology reports, 46(8):38-44, 2010.

[9] V. Chawla AND D. S. HA. An overview of passive rfid. IEEE Communications Magazine, 45(9):11-17, September 2007.

[10] Jiann-Liang Chen, Ming-Chiao Chen, Chien-Wu Chen, and Yao-Chung Chang. Architecture design and performance evaluation of $\{$ RFID $\}$ object tracking systems. Computer Communications, 30(9):2070 - $2086,2007$.

[11] Thomas Chen, Chuang Ta-Tao, and Nakatani Kazuo. The perceived business benefit of cloud computing: An exploratory study. Journal of International Technology and Information Management, 25(4):101 - 121, 2016.

[12] W. T. Chen. An accurate tag estimate method for improving the performance of an rfid anticollision algorithm based on dynamic frame length aloha. IEEE Transactions on Automation Science and Engineering, 6(1):9-15, Jan 2009.

[13] Xun Chen And Z. JAne Wang. Reliable indoor location sensing technique using active rfid. In 2010 The 2nd International Conference on Industrial Mechatronics and Automation, volume 1, pages 160-163, May 2010.

[14] O. Chieochan, A. Saokaew, and E. Boonchieng. An integrated system of applying the use of internet of things, rfid and cloud computing: A case study of logistic management of electricity generation authority of thailand (egat) mae mao lignite coal mining, lampang, thailand. In 2017 9th International Conference on Knowledge and Smart Technology (KST), pages 156-161, Feb 2017.

[15] IBM CorPoration. Ibm perspective on cloud computing, https://www-935.ibm.com/services/in/cio/pdf/ibm_perspective_on _cloud_computing.pdf, November 2016.

[16] Mehdia Ajana El Khaddar, Mohammed Boulmalf, Hamid Harroud, and Mohammed Elkoutbi. Rfid middleware design and architecture. In Designing and Deploying RFID Applications. InTech, 2011.

[17] P. FRIESS. Internet of Things-Global Technological and Societal Trends From Smart Environments and Spaces to Green ICT. River Publishers, 2011.

[18] F. Fuschini, C. Piersanti, L. Sydanheimo, L. Ukkonen, and G. Falciasecca. Electromagnetic analyses of near field uhf rfid systems. IEEE Transactions on Antennas and Propagation, 58(5):1759-1770, May 2010.

[19] G. Garrison, S. Kim, And R. L. WAKEFiEld. Success factors for deploying cloud computing. Communications of the ACM, $55(9): 62-68,2012$.

[20] D. Giusto, Antonio Iera, Giacomo Morabito, And Luigi Atzori. The Internet of Things: 20th Tyrrhenian Workshop on Digital Communications. Springer Science and Business Media, 2010.

[21] A. Gluhak, Srdjan Krco, Michele Nati, Dennis Pfisterer, Nathalie Mitton, and Tahiry Razafindralambo. A survey on facilities for experimental internet of things research. IEEE Communications Magazine, 49(11), 2011.

[22] Y. He AND X. WANG. An aloha-based improved anti-collision algorithm for rfid systems. IEEE Wireless Communications, 20(5):152-158, October 2013.

[23] X. Huang And S. LE. Efficient dynamic framed slotted aloha for rfid passive tags. In The 9th International Conference on Advanced Communication Technology, volume 1, pages 94-97, Feb 2007.

[24] Xu Huang And Son Le. Efficient dynamic framed slotted aloha for rfid passive tags. In Advanced Communication Technology, the 9th International Conference on, volume 1, pages 94-97. IEEE, 2007.

[25] IBM. Ibm perspective on cloud computing, 2011. 
[26] Skyrfid Inc. Reader antenna tutorial - what you need to know. http://skyrfid.com/rfid_antenna_tutorial.php, 2015.

[27] Y. C. LAi AND L. Y. HsiaO. General binary tree protocol for coping with the capture effect in rfid tag identification. IEEE Communications Letters, 14(3):208-210, March 2010.

[28] J. LANDT. The history of rfid. IEEE Potentials, 24(4):8-11, Oct 2005.

[29] Marc Langheinrich. A survey of rfid privacy approaches. Personal and Ubiquitous Computing, 13(6):413-421, 2009.

[30] Shian Liu and Xiaojuan Peng. Improved dynamic frame slotted aloha algorithm for anti-collision in rfid systems. Knowledge Discovery and Data Mining, pages 423-430, 2012.

[31] L. Lu, Y. LIU, AND X. Y. LI. Refresh: Weak privacy model for rfid systems. In 2010 Proceedings IEEE INFOCOM, pages 1-9, March 2010.

[32] Yi-Wei Ma, Wei-Ting Cho, Jiann-Liang Chen, Yueh-Min Huang, and Rongbo Zhu. Rfid-based mobility for seamless personal communication system in cloud computing. Telecommunication Systems, 58(3):233-241, 2015.

[33] C. R Medeiros, Jorge R Costa, and Carlos A Fernandes. Rfid reader antennas for tag detection in self-confined volumes at uhf. IEEE Antennas and Propagation Magazine, 53(2):39-50, 2011.

[34] A. Monzon. Smart cities concept and challenges: Bases for the assessment of smart city projects. Communications in Computer and Information Science, 579:17-31, 2015. cited By 0.

[35] G. Muhammad, S. M. M. Rahman, A. Alelaiwi, and A. Alamri. Smart health solution integrating iot and cloud: A case study of voice pathology monitoring. IEEE Communications Magazine, 55(1):69-73, January 2017.

[36] Husnu S. Narman, Md. Shohrab Hossain, Mohammed Atiquzzaman, and Haiying Shen. Scheduling internet of things applications in cloud computing. Annals of Telecommunications, 72(1):79-93, 2017.

[37] S. Preradovic and N. C. Karmakar. Chipless rfid: Bar code of the future. IEEE Microwave Magazine, 11(7):87-97, Dec 2010.

[38] X. Qing, Z. N. Chen, And C. K. Goh. A uhf near-field/far-field rfid metamaterial-inspired loop antenna. In Proceedings of the 2012 IEEE International Symposium on Antennas and Propagation, pages 1-2, July 2012.

[39] X. Qing, C. K. Goh, And Z. N. CHEN. A broadband uhf near-field rfid antenna. IEEE Transactions on Antennas and Propagation, 58(12):3829-3838, Dec 2010.

[40] Bo RundH. Radio frequency identification (rfid) invaluable technology or a new obstacle in the marketing process? Marketing intelligence $\&$ planning, 26(1):97-114, 2008.

[41] A. Sajid, Haider Abbas, and Kashif Saleem. Cloud-assisted iot-based scada systems security: A review of the state of the art and future challenges. IEEE Access, 4:1375-1384, 2016.

[42] Rifidi Edge Server. Rifidi edge server user's guide.

[43] Transcends-Rifidi Edge Server. Rifidi edge server, http://transcends.co/rifidi-edge-server/, 2016.

[44] Ayob Sether. Cloud computing benefits. Browser Download This Paper, 2016.

[45] S. Shao And R. J. Burkholder. Item-level rfid tag location sensing utilizing reader antenna spatial diversity. IEEE Sensors Journal, 13(10):3767-3774, Oct 2013.

[46] Michael Strasser and Sahin Albayrak. A pattern based feasibility study of cloud computing for smart mobility solutions. In Resilient Networks Design and Modeling (RNDM), 2016 8th International Workshop on, pages 295-301. IEEE, 2016.

[47] W. Su, N. Alchazidis, And T. T. HA. Multiple rfid tags access algorithm. IEEE Transactions on Mobile Computing, $9(2): 174-187$, Feb 2010.

[48] C. C. TAn, B. Sheng, And Q. Li. Secure and serverless rfid authentication and search protocols. IEEE Transactions on Wireless Communications, 7(4):1400-1407, April 2008.

[49] H. Wang, Shuang Chen, and Yong Xie. An rfid-based digital warehouse management system in the tobacco industry: a case study. International Journal of Production Research, 48(9):2513 - 2548, 2010.

[50] R. WANT. An introduction to rfid technology. IEEE pervasive computing, 5(1):25-33, 2006.

[51] R. M. Weber. Internet of things becomes next big thing. Journal of Financial Service Professionals, $70(6): 43-46,2016$.

[52] H. Wu AND Y. Zeng. Passive rfid tag anticollision algorithm for capture effect. IEEE Sensors Journal, 15(1):218-226, Jan 2015.

[53] F. Xia, Laurence T Yang, Lizhe Wang, And Alexey Vinel. Internet of things. International Journal of Communication Systems, 25(9):1101, 2012.

[54] Bo Yan, Chang Yan, Chenxu Ke, and Xingchao Tan. Information sharing in supply chain of agricultural products based on the internet of things. Industrial Management $\&$ Data Systems, 116(7):1397-1416, 2016.

[55] L. YANG, J. HAN, Y. QI, AND Y. LiU. Identification-free batch authentication for rfid tags. In The 18th IEEE International Conference on Network Protocols, pages 154-163, Oct 2010.

[56] D. Zhang, J. Zhou, M. Guo, J. Cao, And T. Li. Tasa: Tag-free activity sensing using rfid tag arrays. IEEE Transactions on Parallel and Distributed Systems, 22(4):558-570, April 2011.

[57] Degan Zhang, Guang Li, Zhaohua Pan, and Yanpin Liang. A new anticollision algorithm for rfid tag. International Journal of Communication Systems, 27(11):3312-3322, 112014.

[58] R. Zhang, Y. LiU, Y. Zhang, And J. Sun. Fast identification of the missing tags in a large rfid system. In 2011 8th Annual IEEE Communications Society Conference on Sensor, Mesh and Ad Hoc Communications and Networks, pages 278-286, June 2011.

Edited by: Kashif Akhtar Saleem

Received: Jul 4, 2017

Accepted: Sep 9, 2017 